Abstract

\title{
Analysis of Generalized Gibbs States ${ }^{\dagger}$
}

\section{Ilya Spitkovsky ${ }^{1}$ and Stephan Weis ${ }^{2}$}

1 New York University Abu Dhabi, Abu Dhabi, United Arab Emirates

2 Independent Researcher, 1050 Bruxelles, Belgium

+ Presented at the Entropy 2021: The Scientific Tool of the 21st Century, 5-7 May 2021; Available online: https://sciforum.net/conference/Entropy2021/.

Published: 5 May 2021

An exponential family is a manifold of generalized Gibbs state of the form $\exp (\mathrm{H}) / \operatorname{Tr}(\exp (\mathrm{H}))$, where $\mathrm{H}$ belongs to a vector space of (possibly non-commutative) hermitian matrices. Generalized Gibbs states are important in small-scale thermodynamics, they represent equilibrium states regarding several conserved quantities that admit novel operations without heat dissipation [1]. Quantum information theory and condensed matter physics consider a space of local Hamiltonians acting on spins. The entropy distance from this exponential family is a measure of many-body complexity [2-4].

This talk is concerned with the geometry and topology of an exponential family and its entropy distance [5]. The maximum-entropy inference map parametrizes the exponential family. This map is continuous in the interior of its domain, the joint numerical range [6]. We describe the points of discontinuity in terms of open mapping theorems and eigenvalue crossings. Because of the discontinuity, the inference map and the entropy distance cannot be approximated through interior points. Instead, it is necessary to study faces (flat portions on the boundary) of the joint numerical range. With local Hamiltonians, this requires studying the faces of the set of quantum marginals.

\section{References}

1. Lostaglio, M.; Jennings, D.; Rudolph, T. Thermodynamic resource theories, non-commutativity and maximum entropy principles. New J. Phys. 2017, 19, 043008.

2. Ay, N.; Olbrich, E.; Bertschinger, N.; Jost, J. A geometric approach to complexity. Chaos 2011, $21,037103$.

3. Niekamp, S.; Galla, T.; Kleinmann, M.; Gühne, O. Computing complexity measures for quantum states based on exponential families. J. Phys. A-Math. Gen. 2013, 46, 125301.

4. Zeng, B.; Chen, X.; Zhou, D.-L.; Wen, X.-G. Quantum Information Meets Quantum Matter; Springer: New York, NY, USA, 2019.

5. Weis, S. Information topologies on non-commutative state spaces. J. Convex Anal. 2014, 21, 339-399.

6. Rodman, L.; Spitkovsky, I.M.; Szkoła, A.; Weis, S. Continuity of the maximum-entropy inference: Convex geometry and numerical ranges approach. J. Math. Phys. 2016, 57, 015204.

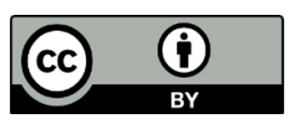

(c) 2021 by the authors. Licensee MDPI, Basel, Switzerland. This article is an open access article distributed under the terms and conditions of the Creative Commons Attribution (CC BY) license (http://creativecommons.org/licenses/by/4.0/). 